\title{
Associations of Education and Income With Secondhand Smoke Exposure Among Non-Smoking Pregnant Women in Japan: The Tohoku Medical Megabank Project Birth and Three-Generation Cohort Study
}

Keiko Murakami ( $\square$ mkeiko-tky@umin.ac.jp )

Tohoku University https://orcid.org/0000-0003-2103-4106

Taku Obara

Tohoku University

Mami Ishikuro

Tohoku Daigaku Global Learning Center

Fumihiko Ueno

Tohoku University

Aoi Noda

Tohoku University

Shinichi Kuriyama

Tohoku University

Research article

Keywords: education, income, Japan, pregnant women, secondhand smoke exposure

Posted Date: September 3rd, 2020

DOI: https://doi.org/10.21203/rs.3.rs-64032/v1

License: (c) (i) This work is licensed under a Creative Commons Attribution 4.0 International License. Read Full License 


\section{Abstract}

Background: Associations of education and income with secondhand smoke (SHS) exposure have been well documented in the general population. However, evidence among non-smoking pregnant women is limited, including in Japan. The purpose of the study was to examine the associations of education and income with SHS exposure among non-smoking pregnant women in Japan.

Methods: We analyzed data from 17815 non-smoking pregnant women in Japan who participated in the Tohoku Medical Megabank Project Birth and Three-Generation Cohort Study from 2013 to 2017. SHS exposure was defined as indoor exposure to someone else's cigarette smoke $\geq 1$ day/week during pregnancy. Multiple logistic regression analyses were conducted to examine whether pregnant women's educational attainment or equivalent household income was associated with SHS exposure, adjusting for age, work status, smoking history, partners' education, and income/education. Stratified analyses by work status were also conducted.

Results: The prevalence of SHS exposure during pregnancy was 34.0\%; 29.4\% among non-working women and $37.1 \%$ among working women. Lower educational attainment was associated with an increased risk of SHS exposure; the odds ratio of high school education or lower compared with university education or higher was 1.78 (95\% confidence interval, 1.59-1.99). Lower equivalent household income was associated with an increased risk of SHS exposure; the odds ratio of the lowest compared with the highest level of income was 1.67 (95\% confidence interval, 1.51-1.84). These associations did not differ between non-working women and working women.

Conclusions: Pregnant women with lower education and/or lower household income had higher risks of SHS exposure in Japan. These findings imply that educational interventions and financial incentives are needed for pregnant women and their household smokers in helping to reduce SHS exposure among non-smoking pregnant women.

\section{Background}

There is growing evidence that secondhand smoke (SHS) exposure during pregnancy has negative consequences for pregnancy and infant outcomes; the 2006 Surgeon General's Report concluded that SHS exposure during pregnancy is causally associated with a reduction in birth weight [1]. More recent studies have demonstrated that SHS exposure during pregnancy is associated with increased risks of stillbirth and congenital anomalies [2, 3]. To design appropriate public health policies, it is important to understand which women are most affected by SHS exposure during pregnancy.

Studies examining the association of education and income with SHS exposure during pregnancy are limited [411], while there is substantial evidence that those with lower education and/or lower income are more exposed to SHS than higher ones in the general population [1, 12-16]. Although education and income reflect the central dimension of social stratification, they have different meanings in society; education is thought to capture knowledge and skills, while income measures material resources to enhance health [17]. Existing studies among pregnant women have mainly focused on education [4, 6, 8-11], and the association between income and SHS exposure have rarely been examined [5, 7]. To the best of our knowledge, no studies have examined the association of education and income with SHS exposure during pregnancy in Japan. Studies in Japan are needed because Japanese people have unique characteristics of tobacco use and SHS exposure. The difference in smoking prevalence between men and women is larger in Japan than in most Organisation for Economic Co- 
operation and Development countries [18]. In addition, World Health Organization (WHO) report in 2015 ranked Japan the lowest among the Framework Convention on Tobacco Control ratifying countries with regard to implementing preventive measures against SHS exposure [19].

Considering the above circumstances, we conducted the present study to examine the associations of education and income with SHS exposure among pregnant women in Japan. In addition, we also examined these associations by work status, because work status is associated with education and income [17] and workplace is one of the major locations for SHS exposure [1].

\section{Methods}

\section{Study population}

Data were obtained from the Tohoku Medical Megabank Project Birth and Three-Generation Cohort Study (TMM BirThree Cohort Study), which has been described elsewhere [20]. Pregnant women and their family members, including the women's partners (the fathers of the fetuses), were contacted in obstetric clinics or hospitals when they scheduled their deliveries from 2013 to 2017. Approximately fifty obstetric clinics and hospitals in Miyagi Prefecture participated in the recruiting process. Tohoku University Tohoku Medical Megabank Organization established seven community support centers in Miyagi Prefecture as local facilities for the voluntary admissiontype recruitment and health assessment of the participants [21]. Trained genome medical research coordinators were placed in each clinic, hospital, or community support center to provide information on the TMM BirThree Cohort Study to potential participants and to receive a signed informed consent form from each participant. Of 32986 pregnant women who were contacted, 22493 agreed to participate in the study and 20879 completed the questionnaires during pregnancy. Among these women, 132 with missing data on smoking status and 631 smokers were excluded. Of the remaining 20116 non-smoking pregnant women, 2301 were excluded because of missing values in equivalent household income, SHS exposure, or work status during pregnancy. The remaining 17815 non-smoking pregnant women were included in the present study. Figure 1 shows the flow diagram of the present study. The TMM BirThree Cohort Study protocol was reviewed and approved by the Ethics Committee of Tohoku University Tohoku Medical Megabank Organization (2013-1-103-1).

\section{Measures}

Pregnant women reported their educational attainment, which were divided into three categories: high school or lower (elementary, junior high school, or senior high school), college (2-year college or special training school), and university or higher (university or graduate school). There were many missing values in educational attainment, partly because this information was collected at one year postpartum. Therefore, a "missing" category was created in addition to these categories. Pregnant women were asked to select their total annual household income from seven categories: $\leq 1.99,2.00-3.99,4.00-5.99,6.00-7.99,8.00-9.99,10.00-11.99$, and $\geq 12.00$ million Japanese yen (JPY). Equivalent household income was calculated as household income divided by the square root of the number of family members [22], and the resulting value was categorized into four groups: $\leq 1.99,2.00-2.99,3.00-$ 3.99 , and $\geq 4.00$ million JPY, which corresponded approximately to quartiles. Work status during pregnancy was measured by how many days per week the women worked. The response of $>0$ days was defined as working.

SHS exposure during pregnancy was measured by how often women reported being exposed to the cigarette smoke of someone else indoors. The responses were almost never, 1 day/week, 2-3 days/week, 4-6 days/week, 
or every day. SHS exposure was defined as 1 day/week or more. This categorization was based on the idea that there is no safe amount of SHS and that breathing even a little SHS can be dangerous [1]. Age was categorized into five groups: $\leq 24,25-29,30-34,35-39$, and $\geq 40$ years. Women's smoking history was categorized as never smoked, quit before becoming aware of the pregnancy, and quit after becoming aware of the pregnancy. If the women's partners participated in the TMM BirThree Cohort Study, they reported their educational attainment on the questionnaire at the time of enrollment. This variable was categorized into the same three groups used to categorize the women's educational attainment.

\section{Statistical analysis}

Multiple logistic regression analyses were conducted to examine the associations of education and income with SHS exposure during pregnancy. We calculated the odds ratios (ORs) and 95\% confidence intervals (Cls) for education and income, adjusted for women's age (model 1), as well as for work status during pregnancy, smoking history, and partner's education (model $2 a$ for education and model $2 b$ for income). We then included income and education simultaneously in the same model (model 3). Variance inflation factors for education and income were within acceptable limits (<2.0), indicating no serious problems of collinearity. We also stratified our models by work status, and examined whether this modified the association by including interaction terms in the models.

All analyses were conducted with SAS version 9.4 (SAS Institute Inc., Cary, NC, USA). For all analyses, a two-tailed $P$ value $<0.05$ was considered statistically significant.

\section{Results}

Table 1 shows the characteristics of non-smoking pregnant women. Prevalence of SHS exposure during pregnancy was $34.0 \%$. A total of $62.0 \%$ was working during pregnancy. Working women were higher educated, had higher equivalent household income, were more likely to be exposed to SHS and never smokers than non-working women. 
Table 1

Characteristics of non-smoking pregnant women: the TMM BirThree Cohort Study

\begin{tabular}{|c|c|c|c|c|c|c|c|}
\hline & \multirow{2}{*}{\multicolumn{2}{|c|}{$\begin{array}{l}\text { Total } \\
(\mathrm{N}=17815)\end{array}$}} & \multicolumn{4}{|c|}{ Work status } & \multirow[t]{3}{*}{$P$-value } \\
\hline & & & \multicolumn{2}{|c|}{$\begin{array}{l}\text { Not working } \\
(n=6771)\end{array}$} & \multicolumn{2}{|c|}{$\begin{array}{l}\text { Working } \\
(n=11044)\end{array}$} & \\
\hline & $\mathbf{n}$ & $(\%)$ & $\mathbf{n}$ & $(\%)$ & $\mathbf{n}$ & $(\%)$ & \\
\hline Educational attainment & & & & & & & $<0.001$ \\
\hline University or higher & 3462 & $(19.4)$ & 1144 & $(16.9)$ & 2318 & $(21.0)$ & \\
\hline College & 4520 & $(25.4)$ & 1725 & $(25.5)$ & 2795 & $(25.3)$ & \\
\hline High school or lower & 3573 & $(20.1)$ & 1646 & $(24.3)$ & 1927 & $(17.5)$ & \\
\hline Missing & 6260 & $(35.1)$ & 2256 & $(33.3)$ & 4004 & $(36.2)$ & \\
\hline Equivalent household income (/year) & & & & & & & $<0.001$ \\
\hline$\geq 4.00$ million Japanese yen & 4605 & $(25.8)$ & 1107 & $(16.4)$ & 3498 & $(31.6)$ & \\
\hline 3.00-3.99 million Japanese yen & 3325 & $(18.7)$ & 1064 & $(15.7)$ & 2261 & $(20.5)$ & \\
\hline 2.00-2.99 million Japanese yen & 5657 & $(31.8)$ & 2500 & $(36.9)$ & 3157 & $(28.6)$ & \\
\hline$\leq 1.99$ million Japanese yen & 4228 & $(23.7)$ & 2100 & $(31.0)$ & 2128 & $(19.3)$ & \\
\hline Secondhand smoke exposure & & & & & & & $<0.001$ \\
\hline Almost never & 11750 & $(66.0)$ & 4798 & $(70.9)$ & 6952 & $(62.9)$ & \\
\hline 1 day/week or more & 6065 & $(34.0)$ & 1973 & $(29.1)$ & 4092 & $(37.0)$ & \\
\hline Age & & & & & & & $<0.001$ \\
\hline$\leq 24$ years & 1273 & $(7.2)$ & 578 & $(8.5)$ & 695 & $(6.3)$ & \\
\hline $25-29$ years & 4739 & $(26.6)$ & 1723 & $(25.5)$ & 3016 & $(27.3)$ & \\
\hline $30-34$ years & 6643 & $(37.3)$ & 2464 & $(36.4)$ & 4179 & $(37.8)$ & \\
\hline $35-39$ years & 4195 & $(23.5)$ & 1599 & $(23.6)$ & 2596 & $(23.5)$ & \\
\hline$\geq 40$ years & 965 & $(5.4)$ & 407 & $(6.0)$ & 558 & $(5.1)$ & \\
\hline Smoking history & & & & & & & $<0.001$ \\
\hline Never smoked & 11063 & $(62.1)$ & 4049 & $(59.8)$ & 7014 & $(63.5)$ & \\
\hline Quit before pregnancy awareness & 4297 & $(24.1)$ & 1774 & $(26.2)$ & 2523 & $(22.8)$ & \\
\hline Quit after pregnancy awareness & 2455 & $(13.8)$ & 948 & $(14.0)$ & 1507 & $(13.7)$ & \\
\hline Partner's educational attainment & & & & & & & $<0.001$ \\
\hline University or higher & 2583 & $(14.5)$ & 1027 & (15.2) & 1556 & (14.1) & \\
\hline College & 1369 & $(7.7)$ & 449 & $(6.6)$ & 920 & $(8.3)$ & \\
\hline
\end{tabular}




\begin{tabular}{|c|c|c|c|c|c|c|c|}
\hline & \multirow{2}{*}{\multicolumn{2}{|c|}{$\begin{array}{l}\text { Total } \\
(\mathrm{N}=17815)\end{array}$}} & \multicolumn{4}{|c|}{ Work status } & \multirow[t]{3}{*}{$P$-value } \\
\hline & & & \multicolumn{2}{|c|}{$\begin{array}{l}\text { Not working } \\
(n=6771)\end{array}$} & \multicolumn{2}{|c|}{$\begin{array}{l}\text { Working } \\
(n=11044)\end{array}$} & \\
\hline & $\mathbf{n}$ & $(\%)$ & $\mathbf{n}$ & $(\%)$ & $\mathbf{n}$ & $(\%)$ & \\
\hline High school or lower & 2403 & $(13.5)$ & 907 & $(13.4)$ & 1496 & $(13.6)$ & \\
\hline Missing & 11460 & $(64.3)$ & 4388 & $(64.8)$ & 7072 & $(64.0)$ & \\
\hline \multicolumn{8}{|c|}{ TMM BirThree Cohort Study, Tohoku Medical Megabank Project Birth and Three-Generation Cohort Study } \\
\hline
\end{tabular}

Table 2 presents the prevalence, ORs and 95\% Cls for SHS exposure during pregnancy. Lower educational attainment was associated with an increased risk of SHS exposure after adjusting for age (model 1) and after additionally adjusting for women's work status, smoking history, and partner's educational attainment (model 2a); the adjusted ORs of high school education or lower compared with university education or higher were 2.50 (95\% $\mathrm{Cl}, 2.25-2.78)$, and $2.00(95 \% \mathrm{Cl}, 1.79-2.23)$, respectively. After further adjustments for equivalent household income, this association was somewhat attenuated but did not disappear; the corresponding OR was $1.78(95 \% \mathrm{Cl}$, 1.59-1.99). Lower equivalent household income was associated with an increased risk of SHS exposure after adjusting for age (model 1) and after additionally adjusting for women's work status, smoking history, and partners' educational attainment (model $2 \mathrm{~b}$ ); the adjusted ORs of $\leq 1.99$ million JPY compared with $\geq 4.00$ million JPY income were 1.96 (95\% Cl, 1.79-2.14), and 1.83 (95\% Cl, 1.67-2.02), respectively. After further adjustments for women's educational attainment, this association was somewhat attenuated but did not disappear; the corresponding OR was $1.67(95 \% \mathrm{Cl}, 1.51-1.84)$. Younger age and working during pregnancy were associated with increased risks of SHS exposure. Women who had quit smoking before pregnancy awareness and women who had quit smoking after pregnancy awareness had increased risks of SHS exposure compared with women who had never smoked: the adjusted ORs were 1.40 (95\% Cl, 1.29-1.51) and 3.08 (95\% Cl, 2.80-3.38), respectively. Having a partner with lower education was associated with an increased risk of SHS exposure; the adjusted OR of high school education or lower compared with university education or higher was $1.61(95 \% \mathrm{Cl}, 1.41-1.83)$. 
Table 2

Associations of education and income with SHS exposure among non-smoking pregnant women

\begin{tabular}{|c|c|c|c|c|c|}
\hline SHS & (\%) & Model 1 & Model 2a & Model 2b & Model 3 \\
\hline /participants & & OR $(95 \% \mathrm{Cl})$ & OR (95\% Cl) & OR $(95 \% \mathrm{Cl})$ & OR $(95 \% \mathrm{Cl})$ \\
\hline
\end{tabular}

Educational

attainment

\begin{tabular}{|c|c|c|c|c|c|c|c|c|}
\hline $\begin{array}{l}\text { University or } \\
\text { higher }\end{array}$ & $781 / 3462$ & $(22.6)$ & 1.00 & & 1.00 & & 1.00 & \\
\hline College & $1412 / 4520$ & (31.2) & 1.55 & $\begin{array}{l}(1.40- \\
1.71)\end{array}$ & 1.35 & $\begin{array}{l}(1.22- \\
1.50)\end{array}$ & 1.27 & $\begin{array}{l}(1.14- \\
1.41)\end{array}$ \\
\hline $\begin{array}{l}\text { High school } \\
\text { or lower }\end{array}$ & $1569 / 3573$ & $(43.9)$ & 2.50 & $\begin{array}{l}(2.25- \\
2.78)\end{array}$ & 2.00 & $\begin{array}{l}(1.79- \\
2.23)\end{array}$ & 1.78 & $\begin{array}{l}(1.59- \\
1.99)\end{array}$ \\
\hline Missing & $2303 / 6260$ & $(36.8)$ & 1.89 & $\begin{array}{l}(1.72- \\
2.08)\end{array}$ & 1.48 & $\begin{array}{l}(1.34- \\
1.64)\end{array}$ & 1.38 & $\begin{array}{l}(1.25- \\
1.52)\end{array}$ \\
\hline
\end{tabular}

Equivalent

household

income

(/year)

\begin{tabular}{|c|c|c|c|c|c|c|c|c|c|c|}
\hline $\begin{array}{l}\geq 4.00 \text { million } \\
\text { Japanese } \\
\text { yen }\end{array}$ & $1215 / 4605$ & $(26.4)$ & 1.00 & & & & 1.00 & & 1.00 & \\
\hline $\begin{array}{l}3.00- \\
3.99 \text { million } \\
\text { Japanese } \\
\text { yen }\end{array}$ & $1010 / 3325$ & (30.4) & 1.17 & $\begin{array}{l}(1.06- \\
1.29)\end{array}$ & & & 1.15 & $\begin{array}{l}(1.03- \\
1.27)\end{array}$ & 1.10 & $\begin{array}{l}(0.99- \\
1.22)\end{array}$ \\
\hline $\begin{array}{l}2.00- \\
2.99 \text { million } \\
\text { Japanese } \\
\text { yen }\end{array}$ & $2010 / 5657$ & (35.5) & 1.45 & $\begin{array}{l}(1.33- \\
1.58)\end{array}$ & & & 1.42 & $\begin{array}{l}(1.29- \\
1.55)\end{array}$ & 1.33 & $\begin{array}{l}(1.22- \\
1.46)\end{array}$ \\
\hline $\begin{array}{l}\leq 1.99 \text { million } \\
\text { Japanese } \\
\text { yen }\end{array}$ & $1830 / 4228$ & (43.3) & 1.96 & $\begin{array}{l}(1.79- \\
2.14)\end{array}$ & & & 1.83 & $\begin{array}{l}(1.67- \\
2.02)\end{array}$ & 1.67 & $\begin{array}{l}(1.51- \\
1.84)\end{array}$ \\
\hline \multicolumn{11}{|l|}{ Age } \\
\hline$\leq 24$ years & $633 / 1273$ & (49.7) & 1.00 & & 1.00 & & 1.00 & & 1.00 & \\
\hline $25-29$ years & $1745 / 4739$ & (36.8) & 0.59 & $\begin{array}{l}(0.52- \\
0.67)\end{array}$ & 0.71 & $\begin{array}{l}(0.62- \\
0.80)\end{array}$ & 0.69 & $\begin{array}{l}(0.61- \\
0.79)\end{array}$ & 0.73 & $\begin{array}{l}(0.64- \\
0.84)\end{array}$ \\
\hline $30-34$ years & $2107 / 6643$ & (31.7) & 0.47 & $\begin{array}{l}(0.42- \\
0.53)\end{array}$ & 0.60 & $\begin{array}{l}(0.53- \\
0.68)\end{array}$ & 0.59 & $\begin{array}{l}(0.52- \\
0.67)\end{array}$ & 0.64 & $\begin{array}{l}(0.56- \\
0.72)\end{array}$ \\
\hline $35-39$ years & $1315 / 4195$ & (31.4) & 0.46 & $\begin{array}{l}(0.41- \\
0.53)\end{array}$ & 0.58 & $\begin{array}{l}(0.51- \\
0.67)\end{array}$ & 0.59 & $\begin{array}{l}(0.51- \\
0.67)\end{array}$ & 0.63 & $\begin{array}{l}(0.55- \\
0.73)\end{array}$ \\
\hline$\geq 40$ years & $265 / 965$ & $(27.5)$ & 0.38 & $\begin{array}{l}(0.32- \\
0.46)\end{array}$ & 0.50 & $\begin{array}{l}(0.41- \\
0.60)\end{array}$ & 0.53 & $\begin{array}{l}(0.44- \\
0.63)\end{array}$ & 0.56 & $\begin{array}{l}(0.46- \\
0.67)\end{array}$ \\
\hline
\end{tabular}




\begin{tabular}{|c|c|c|c|c|c|c|c|c|c|c|}
\hline \multirow{3}{*}{$\begin{array}{l} \\
\begin{array}{l}\text { Work status } \\
\text { during } \\
\text { pregnancy }\end{array}\end{array}$} & \multirow{3}{*}{$\begin{array}{l}\text { SHS } \\
\text { exposure } \\
\text { /participants }\end{array}$} & \multirow[t]{3}{*}{ (\%) } & \multirow{2}{*}{\multicolumn{2}{|c|}{$\begin{array}{l}\text { Model } 1 \\
\text { OR }(95 \% \mathrm{Cl})\end{array}$}} & \multirow{2}{*}{\multicolumn{2}{|c|}{$\begin{array}{l}\text { Model 2a } \\
\text { OR }(95 \% \mathrm{Cl})\end{array}$}} & \multirow{2}{*}{\multicolumn{2}{|c|}{$\begin{array}{l}\text { Model 2b } \\
\text { OR }(95 \% \mathrm{Cl})\end{array}$}} & \multirow{2}{*}{\multicolumn{2}{|c|}{$\begin{array}{l}\text { Model } 3 \\
\text { OR (95\% Cl) }\end{array}$}} \\
\hline & & & & & & & & & & \\
\hline & & & & & & & & & & \\
\hline Not working & $1973 / 6771$ & $(29.1)$ & 1.00 & & 1.00 & & 1.00 & & 1.00 & \\
\hline Working & $4092 / 11044$ & (37.1) & 1.46 & $\begin{array}{l}(1.36- \\
1.56)\end{array}$ & 1.57 & $\begin{array}{l}(1.46- \\
1.68)\end{array}$ & 1.66 & $\begin{array}{l}(1.55- \\
1.78)\end{array}$ & 1.69 & $\begin{array}{l}(1.57- \\
1.81)\end{array}$ \\
\hline \multicolumn{11}{|l|}{$\begin{array}{l}\text { Smoking } \\
\text { history }\end{array}$} \\
\hline $\begin{array}{l}\text { Never } \\
\text { smoked }\end{array}$ & $3071 / 11063$ & $(27.8)$ & 1.00 & & 1.00 & & 1.00 & & 1.00 & \\
\hline $\begin{array}{l}\text { Quit before } \\
\text { pregnancy } \\
\text { awareness }\end{array}$ & $1543 / 4297$ & (35.9) & 1.51 & $\begin{array}{l}(1.40- \\
1.63)\end{array}$ & 1.43 & $\begin{array}{l}(1.32- \\
1.54)\end{array}$ & 1.46 & $\begin{array}{l}(1.35- \\
1.57)\end{array}$ & 1.40 & $\begin{array}{l}(1.29- \\
1.51)\end{array}$ \\
\hline $\begin{array}{l}\text { Quit after } \\
\text { pregnancy } \\
\text { awareness }\end{array}$ & $1451 / 2455$ & $(59.1)$ & 3.59 & $\begin{array}{l}(3.28- \\
3.94)\end{array}$ & 3.18 & $\begin{array}{l}(2.90- \\
3.50)\end{array}$ & 3.25 & $\begin{array}{l}(2.96- \\
3.57)\end{array}$ & 3.08 & $\begin{array}{l}(2.80- \\
3.38)\end{array}$ \\
\hline \multicolumn{11}{|l|}{$\begin{array}{l}\text { Partner's } \\
\text { educational } \\
\text { attainment }\end{array}$} \\
\hline $\begin{array}{l}\text { University or } \\
\text { higher }\end{array}$ & $544 / 2583$ & $(21.1)$ & 1.00 & & 1.00 & & 1.00 & & 1.00 & \\
\hline College & $412 / 1369$ & $(30.1)$ & 1.57 & $\begin{array}{l}(1.35- \\
1.82)\end{array}$ & 1.26 & $\begin{array}{l}(1.08- \\
1.47)\end{array}$ & 1.26 & $\begin{array}{l}(1.08- \\
1.47)\end{array}$ & 1.19 & $\begin{array}{l}(1.02- \\
1.39)\end{array}$ \\
\hline $\begin{array}{l}\text { High school } \\
\text { or lower }\end{array}$ & $967 / 2403$ & $(40.2)$ & 2.38 & $\begin{array}{l}(2.10- \\
2.70)\end{array}$ & 1.74 & $\begin{array}{l}(1.53- \\
1.98)\end{array}$ & 1.78 & $\begin{array}{l}(1.56- \\
2.02)\end{array}$ & 1.61 & $\begin{array}{l}(1.41- \\
1.83)\end{array}$ \\
\hline Missing & $4142 / 11460$ & $(36.1)$ & 2.04 & $\begin{array}{l}(1.84- \\
2.26)\end{array}$ & 1.64 & $\begin{array}{l}(1.48- \\
1.83)\end{array}$ & 1.65 & $\begin{array}{l}(1.48- \\
1.83)\end{array}$ & 1.55 & $\begin{array}{l}(1.39- \\
1.72)\end{array}$ \\
\hline \multicolumn{11}{|c|}{$\mathrm{Cl}$, confidence interval; OR, odds ratio; SHS, secondhand smoke. } \\
\hline \multicolumn{11}{|c|}{ Model 1: adjusted for age. } \\
\hline \multicolumn{11}{|c|}{$\begin{array}{l}\text { Model } 2 \mathrm{a} \text { (for education) and } 2 \mathrm{~b} \text { (for income): model } 1+\text { adjusted for work status during pregnancy, smoking } \\
\text { history, and partner's educational attainment. }\end{array}$} \\
\hline
\end{tabular}

Table 3 presents the results of the analyses stratified by work status. Lower educational attainment was associated with an increased risk of SHS exposure both among non-working women and working women; the adjusted ORs of high school education or lower compared with university education or higher were $1.76(95 \% \mathrm{Cl}$, $1.45-2.14)$, and $1.79(95 \% \mathrm{Cl}, 1.56-2.06)$, respectively (model 3$)$. No significant interaction was found between work status and educational attainment $(P=0.06)$. Lower equivalent household income was associated with an increased risk of SHS exposure both among non-working women and working women; the adjusted ORs of $\leq$ 1.99 million JPY compared with $\geq 4.00$ million JPY income were $1.47(95 \% \mathrm{Cl}, 1.22-1.76)$, and $1.78(95 \% \mathrm{Cl}, 1.58-$ 
2.01), respectively (model 3). No significant interaction was found between work status and equivalent household income $(P=0.35)$. 
Table 3

Associations of education and income with SHS exposure by work status among non-smoking pregnant women

\begin{tabular}{llllll}
$\begin{array}{l}\text { SHS } \\
\text { exposure }\end{array}$ & $(\%)$ & Model 1 & Model 2a & Model 2b & Model 3 \\
\cline { 2 - 5 } /participants & & OR $(95 \% \mathrm{Cl})$ & OR $(95 \% \mathrm{Cl})$ & OR (95\% Cl) & OR (95\% Cl)
\end{tabular}

Educational

attainment

Not working

$(n=6771)$

$\begin{array}{lllll}\text { University or } & 205 / 1144 & (17.9) & 1.00 & 1.00\end{array}$

higher

\begin{tabular}{|c|c|c|c|c|c|c|c|c|}
\hline College & $410 / 1725$ & $(23.8)$ & 1.43 & $\begin{array}{l}(1.18- \\
1.72)\end{array}$ & 1.25 & $\begin{array}{l}(1.03- \\
1.51)\end{array}$ & 1.20 & $\begin{array}{l}(0.98- \\
1.46)\end{array}$ \\
\hline $\begin{array}{l}\text { High school } \\
\text { or lower }\end{array}$ & $632 / 1646$ & (38.4) & 2.58 & $\begin{array}{l}(2.15- \\
3.10)\end{array}$ & 1.88 & $\begin{array}{l}(1.55- \\
2.28)\end{array}$ & 1.76 & $\begin{array}{l}(1.45- \\
2.14)\end{array}$ \\
\hline Missing & $726 / 2256$ & (32.2) & 2.00 & $\begin{array}{l}(1.68- \\
2.39)\end{array}$ & 1.50 & $\begin{array}{l}(1.25- \\
1.81)\end{array}$ & 1.44 & $\begin{array}{l}(1.20- \\
1.73)\end{array}$ \\
\hline
\end{tabular}

Working $(\mathrm{n}=$

11044)

\begin{tabular}{|c|c|c|c|c|c|c|c|c|}
\hline $\begin{array}{l}\text { University or } \\
\text { higher }\end{array}$ & $576 / 2318$ & $(24.9)$ & 1.00 & & 1.00 & & 1.00 & \\
\hline College & $1002 / 2795$ & (35.9) & 1.67 & $\begin{array}{l}(1.48- \\
1.89)\end{array}$ & 1.42 & $\begin{array}{l}(1.25- \\
1.61)\end{array}$ & 1.31 & $\begin{array}{l}(1.15- \\
1.49)\end{array}$ \\
\hline $\begin{array}{l}\text { High school } \\
\text { or lower }\end{array}$ & $937 / 1927$ & $(48.6)$ & 2.71 & $\begin{array}{l}(2.37- \\
3.09)\end{array}$ & 2.08 & $\begin{array}{l}(1.81- \\
2.38)\end{array}$ & 1.79 & $\begin{array}{l}(1.56- \\
2.06)\end{array}$ \\
\hline Missing & $1577 / 4004$ & $(39.4)$ & 1.88 & $\begin{array}{l}(1.68- \\
2.11)\end{array}$ & 1.48 & $\begin{array}{l}(1.31- \\
1.66)\end{array}$ & 1.35 & $\begin{array}{l}(1.20- \\
1.53)\end{array}$ \\
\hline
\end{tabular}

Equivalent

household

income

(/year)

Not working

$(\mathrm{n}=6771)$

\begin{tabular}{|c|c|c|c|c|c|c|c|c|}
\hline $\begin{array}{l}\geq 4.00 \text { million } \\
\text { Japanese } \\
\text { yen }\end{array}$ & $231 / 1107$ & (20.9) & 1.00 & & 1.00 & & 1.00 & \\
\hline $\begin{array}{l}3.00- \\
3.99 \text { million } \\
\text { Japanese } \\
\text { yen }\end{array}$ & $242 / 1064$ & $(22.7)$ & 1.05 & $\begin{array}{l}(0.85- \\
1.29)\end{array}$ & 1.02 & $\begin{array}{l}(0.83- \\
1.26)\end{array}$ & 1.01 & $\begin{array}{l}(0.82- \\
1.25)\end{array}$ \\
\hline $\begin{array}{l}2.00- \\
2.99 \text { million } \\
\text { Japanese } \\
\text { yen }\end{array}$ & $731 / 2500$ & (29.2) & 1.42 & $\begin{array}{l}(1.20- \\
1.69)\end{array}$ & 1.29 & $\begin{array}{l}(1.08- \\
1.54)\end{array}$ & 1.25 & $\begin{array}{l}(1.05- \\
1.49)\end{array}$ \\
\hline
\end{tabular}




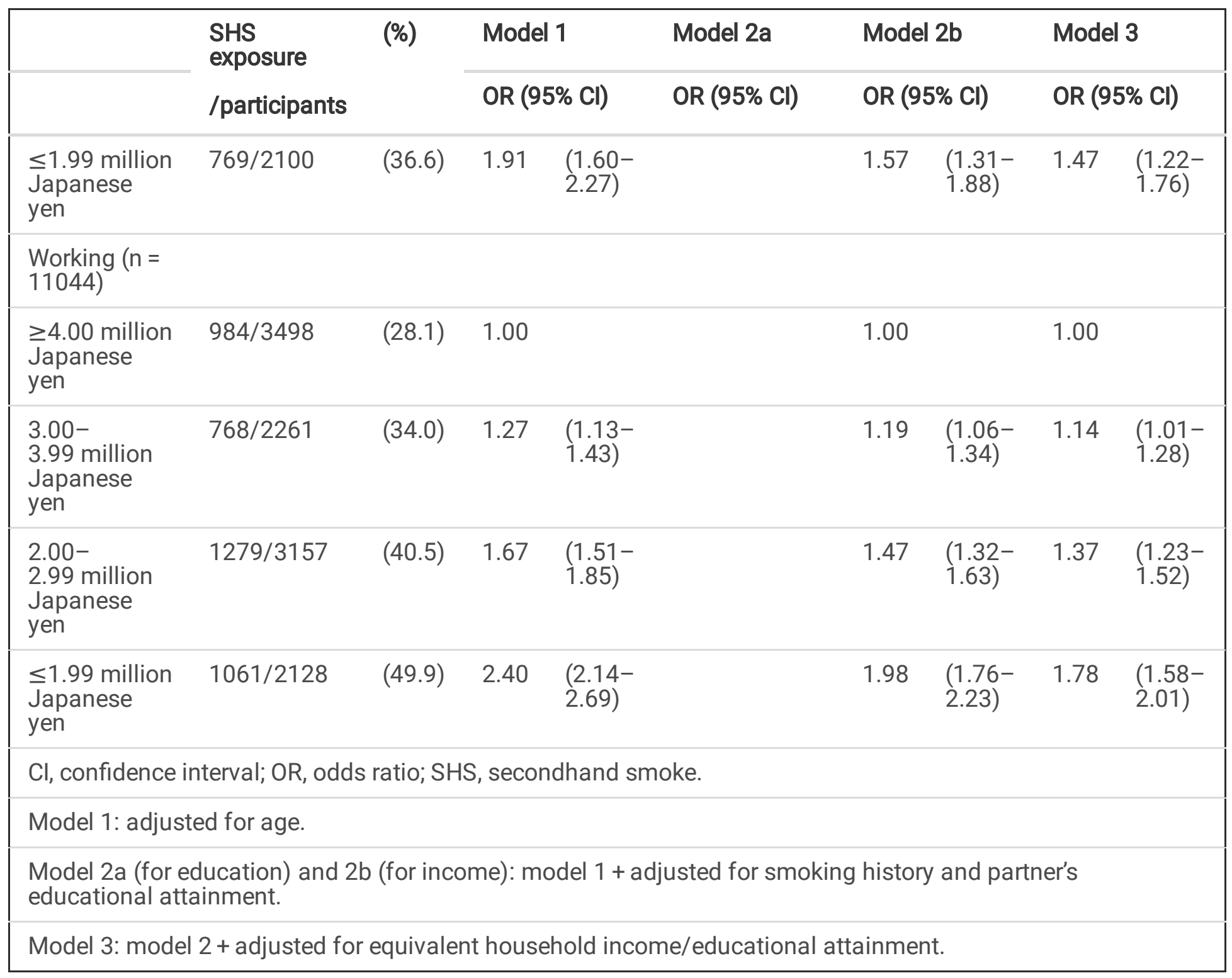

\section{Discussion}

The present study examined the associations of education and income with SHS exposure among pregnant women in Japan. About one-third of pregnant women were exposed to SHS. Pregnant women with lower educational attainment and/or lower equivalent household income had significantly higher risks of SHS exposure than pregnant women with higher education and/or income. These associations did not differ between nonworking women and working women.

Lower education was associated with an increased risk of SHS exposure during pregnancy, independent of equivalent household income. There are several possible explanations for this association. First, education conveys factual health-related knowledge [23], and lower educated people working jobs offering few opportunities for learning may have limited knowledge of the harmful effects of unhealthy behaviors [24]. WHO guidelines recommend that health care providers should provide pregnant women with advice and information about the risks of SHS exposure [25], reflecting the importance of knowledge of the risks of SHS exposure among pregnant women. Second, education raises cognitive skills that foster health-promoting decisions [23, 26]. Hence, education may increase individuals' understanding of the negative effects of SHS exposure and build their capacity to 
manage their own SHS exposure [24]. Women's capacity to manage their SHS exposure is affected by the intersecting influences of gender roles and power [27]. A small number of clinical interventions have suggested that providing brief advice or counseling to non-smoking pregnant women may reduce their SHS exposure [28]. Third, education increases the chances of having a well-educated partner [23]. In Japan, men with lower education have been found to have a higher risk of current smoking, especially among the young population [29], and SHS from partners is a major source of exposure for non-smoking women [6]. However, the present study showed that women's education was associated with SHS exposure after adjusting for partner's education. Fourth, social networks, which combine individuals' resources with those of others [30], may also partially explain the observed association. Having a higher level of education increases the chances of associating with other highly educated individuals, and social networks made up of such individuals communicate health-promoting behaviors, thus widening disparities by educational attainment $[23,24,30]$. Higher levels of education also correspond to a greater likelihood of friends emulating each other's smoking behavior [31], which could lead to an association between education and SHS exposure.

We also found that lower equivalent household income was associated with an increased risk of SHS exposure during pregnancy, independent of education. This association was observed among non-working women as well as among working women. This result suggests that the partners and other household members of pregnant women with lower equivalent household income might be more likely to smoke, compared with those with higher equivalent household income. The association between lower income and smoking has been consistently shown [32], including among community-dwelling people in Japan [33]. One possible mechanism by which lower income has been hypothesized to be associated with smoking is that people with lower income are more susceptible to the tobacco industry's marketing strategy of glamorizing smoking by associating it with wealth and success [32]. Banning advertising that gives the impression that smoking is attractive might be useful, although no studies have examined the impact of advertising bans on the association between income and smoking [34]. A higher prevalence of smoking among lower-income individuals may also be explained by several other factors such as the frequent normalization of smoking and earlier smoking initiation, less concern about harm caused by smoking, poorer access to smoking cessation resources, and more difficulty with successfully quitting among this group [32, 34]. Adopting many health behaviors does not require money, but paying for smoking cessation aids such as individual counseling and medications can help individuals to realize their desire to quit $[23,24]$. According to WHO guidelines, health care providers should give partners and other household members advice and information about the risks to pregnant women from SHS exposure and, if possible, also provide them with cessation support [25]. Effective interventions to create smoke-free homes alongside smoking cessation for them, perhaps involving financial incentives, need to be developed $[35,36]$. On the other hand, we also found that working women, who had higher equivalent household income, were more likely to be exposed to SHS than were non-working women. This suggests that measures against SHS exposure at work, such as totally smoke-free workplace policies, should also be implemented [37].

To our knowledge, this is the first study to demonstrate the associations of education and income with SHS exposure during pregnancy in Japan. By examining education and income simultaneously, we showed independent associations between SHS exposure and both education and income, which have different meanings in society [17]. Although Japan lagged behind Western countries in terms of preventive measures against SHS exposure [16], the Japanese government approved its first national smoking ban for public facilities in July 2018, and this ordinance has come into force in April 2020. This measure is expected to be beneficial for reducing SHS exposure for non-smoking pregnant women [38]. Nevertheless, the association of education and income with SHS

Page $12 / 17$ 
exposure in Japan should continue to be monitored after the introduction of this national smoking ban, because smaller magnitudes of declines in SHS exposure for lower socioeconomic groups than for higher socioeconomic groups have been found to increase the disparity in SHS exposure between these groups in Western countries [14].

The present study has several limitations. First, about one-third of the analytic women had missing values in their educational attainment. As they were more likely to be exposed to SHS than those with university education or higher, the observed associations in the present study may have underestimated the true associations between education and SHS exposure. Second, this study was conducted at one of 47 prefectures in Japan; therefore, the generalizability of the present study is limited. However, there is little difference between the prevalence of smoking in Miyagi Prefecture and that reported in a national survey in 2016: 33.4\% among men and $9.7 \%$ among women in Miyagi Prefecture and 31.1\% among men and 9.5\% among women in the national survey [39]. Finally, SHS exposure was self-reported. It is possible that some women underreported active smoking and also SHS exposure because active smoking has become more socially unacceptable [40,41], again leading to an underestimation of the association.

\section{Conclusions}

Women with lower education and/or lower income had higher risks of SHS exposure during pregnancy in Japan. These associations did not differ between non-working women and working women. These findings imply that educational interventions and financial incentives are needed for pregnant women and their household smokers in helping to reduce SHS exposure among non-smoking pregnant women.

\section{List Of Abbreviations}

95\% Cl: 95\% confidence interval; OR: odds ratio; TMM BirThree Cohort Study: Tohoku Medical Megabank Project Birth and Three-Generation Cohort Study

\section{Declarations}

\section{Ethics approval and consent to participate}

The TMM BirThree Cohort Study protocol was reviewed and approved by the Ethics Committee of Tohoku University Tohoku Medical Megabank Organization (2013-1-103-1). Trained genome medical research coordinators were placed in each clinic, hospital, or community support center to provide information on the TMM BirThree Cohort Study to potential participants and to receive signed informed consent forms from those who agreed to participate.

\section{Consent for publication}

There are no details on individual participants within the manuscript.

\section{Availability of data and material}

A biobank is being constructed based on the TMM BirThree Cohort Study. Although we are planning to share the full baseline data by the end of 2020, a portion of the data have been distributed to researchers who have been approved by the Sample and Data Access Committee of the Biobank since 2017. 


\section{Competing interests}

The authors declare that they have no competing interests.

\section{Funding}

The TMM BirThree Cohort Study was supported by the Japan Agency for Medical Research and Development (AMED), Japan [grant number, JP19km0105001]. KM was supported by a Grant-in-Aid for Early-Career Scientists (No. 18K17397) from the Ministry of Education, Culture, Sports, Science and Technology, Japan.

\section{Authors' contributions}

KM was responsible for the study conception, design, analysis, and interpretation of the data, as well as the drafting of the manuscript. SK provided advice regarding critically important intellectual content and helped to draft the manuscript. All authors acquired the data and intensively revised the manuscript.

\section{Acknowledgements}

The authors wish to express their appreciation to the pregnant women who participated in the TMM BirThree Cohort Study.

\section{References}

1. S. Department of Health and Human Services. The Health Consequences of Involuntary Exposure to Tobacco Smoke: A Report of the Surgeon General.Atlanta, GA: U.S. Department of Health and Human Services, Centers for Disease Control and Prevention, Coordinating Center for Health Promotion, National Center for Chronic Disease Prevention and Health Promotion, Office on Smoking and Health; 2006.

2. Salmasi G, Grady R, Jones J, McDonald SD; Knowledge Synthesis Group. Environmental tobacco smoke exposure and perinatal outcomes: a systematic review and meta-analyses. Acta Obstet Gynecol Scand. 2010;89:423-

3. Leonardi-Bee J, Britton J, Venn A. Secondhand smoke and adverse fetal outcomes in nonsmoking pregnant women: a meta-analysis. Pediatrics. 2011;127:734-

4. Vardavas $\mathrm{Cl}$, Patelarou $\mathrm{E}$, Chatzi L, et al. Factors associated with active smoking, quitting, and secondhand smoke exposure among pregnant women in Greece. J Epidemiol. 2010;20:355-

5. Yoo SH, Paek YJ, Kim SS, et al. Hair nicotine levels in non-smoking pregnant women whose spouses smoke outside of the home. Tob Control. 2010;19:318-

6. Aurrekoetxea JJ, Murcia M, Rebagliato M, et al. Factors associated with second-hand smoke exposure in nonsmoking pregnant women in Spain: self-reported exposure and urinary cotinine levels. Sci Total Environ. 2014;470-471:1189-

7. Hawkins SS, Dacey C, Gennaro S, et al. Secondhand smoke exposure among nonsmoking pregnant women in New York City. Nicotine Tob Res. 2014;16:1079-

8. Polanska K, Krol A, Kaluzny P, Ligocka D, Mikolajewska K, Shaheen S, Walton R, Hanke W. Estimation of saliva cotinine cut-off points for active and passive smoking during pregnancy - Polish Mother and Child Cohort (REPRO_PL). Int J Environ Res Public Health. 2016;13:1216. 
9. Hikita N, Haruna M, Matsuzaki M, Sasagawa E, Murata M, Oidovsuren O, Yura A. Prevalence and risk factors of secondhand smoke (SHS) exposure among pregnant women in Mongolia. Sci Rep. 2017;7:16426.

10. Do EK, Green TL, Prom-Wormley EC, et al. Social determinants of smoke exposure during pregnancy: findings from waves $1 \& 2$ of the Population Assessment of Tobacco and Health (PATH) Study. Prev Med Rep. 2018;12:312-

11. Reece S, Morgan C, Parascandola M, Siddiqi K. Secondhand smoke exposure during pregnancy: a crosssectional analysis of data from Demographic and Health Survey from 30 low-income and middle-income countries. Tob Control. 2019;28:420-

12. World Health Organization. Environmental Health Inequalities in Europe. Assessment Report. Geneva: World Health Organization; 2012.

http://www.euro.who.int/en/publications/abstracts/environmental-health-inequalities-in-europe.-assessmentreport. Accessed 14 Aug 2020.

13. Tabuchi T, Nakamura M. [Disparity of secondhand smoke exposure at home and/or workplace according to age, education and medical insurance in Japan]. JACR Monograph. 2014;20:39-48 (in Japanese; Abstract in English).

14. Gan WQ, Mannino DM, Jemal A. Socioeconomic disparities in secondhand smoke exposure among US neversmoking adults: the National Health and Nutrition Examination Survey 1988-2010. Tob Control. 2015;24:568-

15. Matsuyama Y, Aida J, Tsuboya T, et al. Social inequalities in secondhand smoke among Japanese nonsmokers: a cross-sectional study. J Epidemiol. 2018;28:133-

16. Nguyen M, Nishi N, Kadota A, et al. Passive smoking at home by socioeconomic factors in a Japanese population: NIPPON DATA2010. J Epidemiol. 2018;28 Suppl 3:S40-4

17. Galobardes B, Shaw M, Lawlor DA, et al. Indicators of socioeconomic position. In: Oakes JM, Kaufman JS, editors. Methods in Social Epidemiology. San Francisco, CA: Jossey-Bass; 2006:47-85.

18. Organisation for Economic Co-operation and Development. OECD Reviews of Public Health: Japan: A Healthier Tomorrow. Paris, France: OECD Publishing; 2019.

https://doi.org/10.1787/9789264311602-en. Published February 2019. Accessed 14 Aug 2020.

19. World Health Organization. WHO Report on the Global Tobacco Epidemic 2015: Raising Taxes on Tobacco. Geneva: World Health Organization;

20. Kuriyama S, Metoki H, Kikuya M, et al. Cohort Profile: Tohoku Medical Megabank Project Birth and ThreeGeneration Cohort Study (TMM BirThree Cohort Study): rationale, progress and perspective. Int J Epidemiol. 2020;49:18-

21. Kuriyama S, Yaegashi N, Nagami F, et al. The Tohoku Medical Megabank Project: design and mission. J Epidemiol. 2016;26:493-

22. Atkinson, AB, Rainwater L, Smeeding TM. Income Distribution in OECD Countries: Evidence from the Luxembourg Income Study. Paris, France: Organization for Economic Co-operation and Development; 1995.

23. Glymour MM, Avendano M, Kawachi I. Socioeconomic status and health. In: Berkman LF, Kawachi I, Glymour MM, eds. Social Epidemiology. New York, NY: Oxford University Press; 2014:17-62.

24. Pampel FC, Krueger PM, Denney JT. Socioeconomic Disparities in Health Behaviors. Annu Rev Sociol. 2010;36:349-70.

25. World Health Organization. WHO recommendations for the prevention and management of tobacco use and second-hand smoke exposure in pregnancy. Geneva: World Health Organization; 2013. 
http://www.who.int/tobacco/publications/pregnancy/guidelinestobaccosmokeexposure/en/. Accessed 14 Aug 2020.

26. Cutler DM, Lleras-Muney A. Understanding differences in health behaviors by education. J Health Econ. 2010;29:1-

27. Greaves LJ, Hemsing NJ. Sex, gender, and secondhand smoke policies: implications for disadvantaged women. Am J Prev Med. 2009;37(2 Suppl):S131-S13

28. Tong VT, Dietz PM, Rolle IV, Kennedy SM, Thomas W, England LJ. Clinical interventions to reduce secondhand smoke exposure among pregnant women: a systematic review. Tob Control. 2015;24:217-

29. Tabuchi T, Kondo N. Educational inequalities in smoking among Japanese adults aged 25-94 years: Nationally representative sex- and age-specific statistics. J Epidemiol. 2017;27:186-

30. Berkman LF, Krishna A. Social network epidemiology. In: Berkman LF, Kawachi I, Glymour MM, eds. Social Epidemiology. New York, NY: Oxford University Press; 2014:234-289.

31. Christakis NA, Fowler JH. The collective dynamics of smoking in a large social network. N Engl J Med. 2008;358:2249-

32. Casetta B, Videla AJ, Bardach A, et al. Association between cigarette smoking prevalence and income level: a systematic review and meta-analysis. Nicotine Tob Res. 2017;19:1401-

33. Fukuda Y, Nakamura K, Takano T. Socioeconomic pattern of smoking in Japan: income inequality and gender and age differences. Ann Epidemiol 2005;15:365-

34. Hill S, Amos A, Clifford D, et al. Impact of tobacco control interventions on socioeconomic inequalities in smoking: review of the evidence. Tob Control. 2014;23(e2):e89-

35. Dherani M, Zehra SN, Jackson C, Satyanaryana V, Huque R, Chandra P, Rahman A, Siddiqi K. Behaviour change interventions to reduce second-hand smoke exposure at home in pregnant women - a systematic review and intervention appraisal. BMC Pregnancy Childbirth. 2017;17:378.

36. Nwosu C, Angus K, Cheeseman H, Semple S. Reducing second-hand smoke exposure among non-smoking pregnant women: a systematic review [published online ahead of print May 19, 2020]. Nicotine Tob Res. doi:10.1093/ntr/ntaa089.

37. Fichtenberg CM, Glantz SA. Effect of smoke-free workplaces on smoking behaviour: systematic review. BMJ. 2002;325(7357):188.

38. Schechter JC, Fuemmeler BF, Hoyo C, Murphy SK, Zhang JJ, Kollins SH. Impact of smoking ban on passive smoke exposure in pregnant non-smokers in the southeastern United States. Int J Environ Res Public Health. 2018;15:83.

39. Ministry of Health, Labour and Welfare. Comprehensive Survey of Living Conditions in Japan, 2016. https://www.mhlw.go.jp/toukei/saikin/hw/k-tyosa/k-tyosa16/. Accessed 14 Aug 2020.

40. Shipton D, Tappin DM, Vadiveloo T, Crossley JA, Aitken DA, Chalmers J. Reliability of self reported smoking status by pregnant women for estimating smoking prevalence: a retrospective, cross sectional study. BMJ. 2009;339:b4347.

41. Sasaki S, Braimoh TS, Yila TA, Yoshioka E, Kishi R. Self-reported tobacco smoke exposure and plasma cotinine levels during pregnancy-a validation study in Northern Japan. Sci Total Environ. 2011;412-413:114-

\section{Figures}




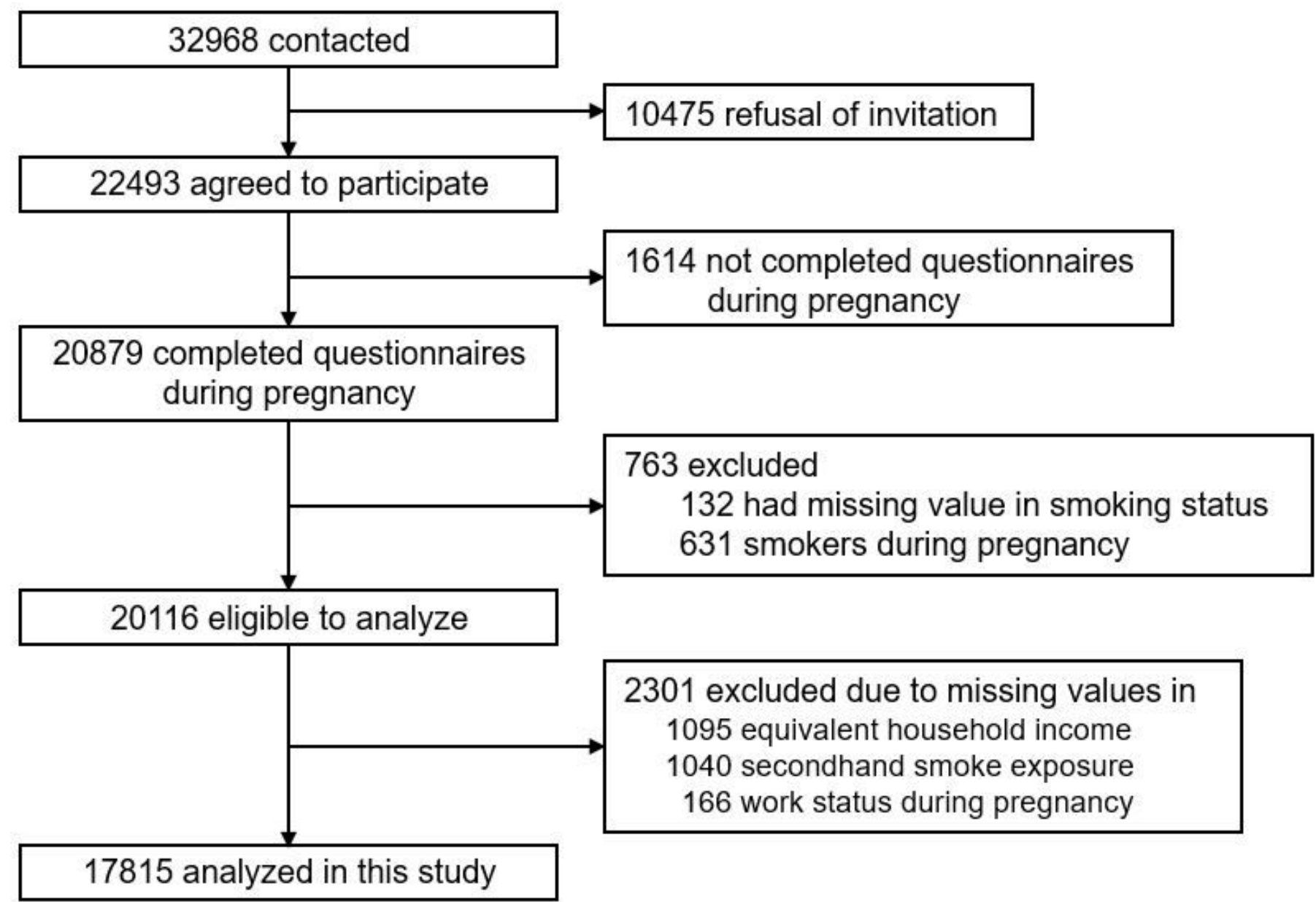

Figure 1

Flow diagram of participants in the present analysis of the TMM BirThree Cohort Studya aTohoku Medical Megabank Project Birth and Three-Generation Cohort Study 